\title{
Tapered Liquid-Core All-Fibre Devices for Low-Threshold Raman Generation
}

\author{
Limin Xiao*, N. Healy, and A. C. Peacock \\ Optoelectronics Research Centre, University of Southampton, Southampton, SO17 1BJ, UK \\ “lx1v10@orc.soton.ac.uk
}

Liquid-core fibres (LCF) which combine long interaction lengths and strong field confinement to enhance the nonlinear fluid-optic interactions are currently attracting much interest [1, 2]. The resurgence in this field is in part due to the ever increasing array of fibre templates (principally the microstructured designs) in which to infiltrate the materials, but also due to recent efforts to fully integrate the LCFs with standard single mode fibres (SMF) [1], thus improving the efficiency as well as the robustness of the devices. An extension to this work which allows for further enhancement of the nonlinear processes, as well as tailoring of the dispersion properties, is to employ tapered hollow core fibre (HCF) designs. Here we present the first results on the fabrication and characterization of a tapered liquid-core all-fibre optical device (TLC-AFOD) and subsequently demonstrate its use for low threshold stimulated Raman scattering in toluene.

The tapered HCF used in our investigations had a starting inner diameter of $5 \mu \mathrm{m}$ and was tapered using a Vytran GPX-3000 glass processing system to an internal waist of $2 \mu \mathrm{m}$ (Fig. 1 (a)). The total HCF length was 20 $\mathrm{cm}$, with the tapered waist over $7.5 \mathrm{~cm}$ and transition lengths of $\sim 1 \mathrm{~cm}$. As shown in Fig. 1(b), the two ends of the tapered HCF were spliced to angle-cleaved $\left(6^{\circ} \sim 8^{\circ}\right)$ SMF-28 without collapsing the air-holes. This allowed for the liquid to be infiltrated without any bubbles at the end interfaces, as illustrated by the minimal scattering of light across the joint in Fig.1 (c). Here the toluene was incorporated using the capillary action by placing a liquid filled vial on one of the splice joints. Following complete filling, a second vial was placed on the remaining joint, and similarly filled with toluene to avoid evaporation [1]. The total device loss is $\sim 5 \mathrm{~dB}$ at 532 $\mathrm{nm}$, which includes the loss of toluene, the mode mismatch between the SMF and the smaller core capillary at the two joints, as well as some minor scattering. Comparing this with the loss of an untapered device of the same length, we estimate the loss introduced by the taper to be $\sim 0.2 \mathrm{~dB}$.
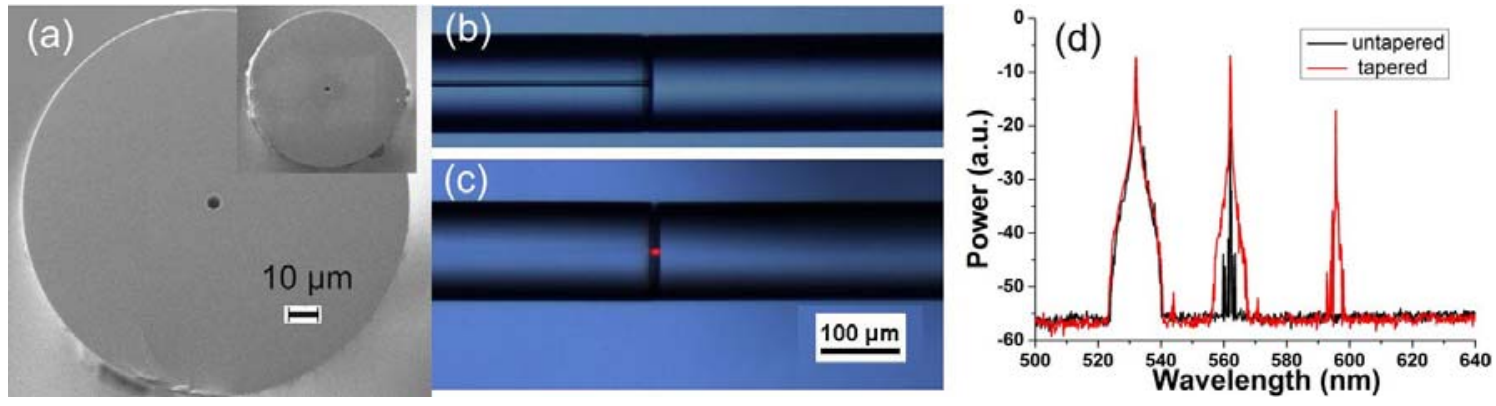

Fig. 1 (a) Scanning electron micrograph of the cross sections of $5 \mu \mathrm{m}$ core HCF and tapered $2 \mu \mathrm{m}$ core (inset). (b) Gap splicing joint between $5 \mu \mathrm{m}$ core HCF (left) and SMF-28 (right). (c) Illustration of guided 633nm light in a toluene filled core showing minimal scattered light at the interface. (d) Raman spectra at $35 \mu \mathrm{w}$ pump power; the first Stokes line is at $562 \mathrm{~nm}$, the second is at $595 \mathrm{~nm}$.

A frequency doubled Nd:YVO 4 Q-switched pulsed green laser $(532 \mathrm{~nm}, 10 \mathrm{~ns}, 20 \mathrm{~Hz}$ ) was used to pump the TLC-AFOD, and the threshold of the generated Stokes wave was measured to be as low as $18 \mu \mathrm{W}$. When the pump power is increased to $35 \mu \mathrm{W}$, we observe cascading to the second Stokes wave, as shown in Fig. 1(d) (red curve). Comparing this with the untapered device pumped at the same power (black curve) where only the first Stokes wave is generated, we estimate that the Raman threshold of the TLC-AFOD to be around two times lower, consistent with the theoretical estimation. To demonstrate the flexibility of this approach, we have also fabricated a TLC-AFOD with a core waist down to $300 \mathrm{~nm}$, which should be suitable for infiltration with high index liquids such as carbon disulphide for even tighter mode confinement. We anticipate that by using liquids with higher linear and nonlinear refractive indices that we can continue to reduce the nonlinear thresholds in such devices for low-threshold lasing, supercontinuum, and pulse shaping etc.

In summary, these TLC-AFOD fibres offer a number of advantages in that they are robust (avoid evaporation), compact, and fully integrated, with the potential to tailor both the dispersion and nonlinearity. We believe that this work will open up many new nonlinear applications of liquid-core fibres.

\section{References}

[1] K. Kieu, L. Schneebeli, R. A. Norwood, and N. Peyghambarian, "Integrated liquid-core optical fibers for ultra-efficient nonlinear liquid photonics," Opt. Express 20, 8148-8154 (2012).

[2] Y.H. Xu, X. F. Chen, and Y. Zhu, "Modeling of micro-diameter-scale liquid core optical fiber filled with various liquids," Opt. Express 16, 9205-9212 (2008). 